\title{
Descifrando los niveles de integración de los sistemas integrados de gestión
}

\section{Deciphering the levels of integration of the integrated management systems}

Alexandra Bonilla Palacios**

Montajes Morelco S.A

Jorge Martine* García****

Corporación Andina de estudios técnicos y desarrollo empresarial

\section{RESUMEN}

En el siguiente artículo se presenta un mecanismo de medición que tiene como propósito medir niveles de integración de los sistemas de gestión ISO 9001, ISO 14001 y OHSAS 18001 implementados dentro de las organizaciones. Se realiza un análisis bibliográfico de los sistemas y las metodologías para integrarlos, dentro del cual se referencian teorías y normas creadas para tal propósito. Se determina un concepto de integración y una escala de niveles de integración alineada al concepto de integración seleccionado. A partir de esto se crea una herramienta que al diagnosticar los niveles de integración de una organización, permite tomar acciones pertinentes para aprovechar los beneficios que trae para las organizaciones el hecho de tener un sistema integrado.

Palabras clave: calidad, sistemas integrados, niveles de integración.

\footnotetext{
* Artículo resultado de investigación.
}

** Ingeniera Agroindustrial y magíster en Calidad y Gestión Integral. Coordinadora Nacional de Calidad en Montajes Morelco S.A. Correo electrónico: alebonillap@gmail.com.

*** Administrador de Empresas y magíster en Calidad y Gestión Integral. Docente en la Corporación Andina de Estudios Técnicos y Desarrollo Empresarial. Correo electrónico: jueayanami@hotmail.com. 


\section{ABSTRACT}

This article presents a mechanism that aims to measure levels of integration levels of standardized management systems ISO 9001, ISO 14001 and OSHAS 18001 implemented into organizations. For that start to make an analysis of systems and methodologies for integrate within are referenced theories and standards created for that purpose. Starting by unique concept and scale levels of aligned integration to the concept select integration created one tool that to diagnose the levels at within organization is permitted take appropriate actions for reap the benefits it brings to the organizations has an integrated system.

Keywords: quality, integrated systems, integration levels.

\section{INTRODUCCIÓN}

Los sistemas de gestión se vinculan en la configuración de las estrategias globales de las organizaciones y alrededor de estos sistemas se pueden construir ventajas competitivas en el mercado, tanto a nivel de producto como de procesos. Así es como al integrar los sistemas de gestión se maximiza el aprovechamiento de estas ventajas para las organizaciones.

Actualmente existen normas técnicas para estandarizar sistemas de gestión que se enfocan en aspectos específicos del funcionamiento de las empresas, como son la calidad, el ambiente y la salud y seguridad de los trabajadores, en las cuales las empresas se certifican para lograr mayor eficacia de sus actividades.

Cuando las empresas implementan más de un sistema de gestión, la estructura con la que inicialmente son creados estos estándares permite que se puedan integrar unos con otros. En este sentido, cuanto mayor sea el grado de integración, más se aprovecha el potencial que ofrecen los sistemas de gestión para conseguir los objetivos organizacionales.

Determinar el nivel de integración de los sistemas de gestión es clave al momento de tomar acciones para su posterior gestión, pero en la actualidad no existe un mecanismo que permita medir el nivel de integración de sistemas de gestión. Esto imposibilita obtener diagnósticos precisos acerca de los aspectos en los cuales se debe enfocar el mayor esfuerzo de la gestión para aprovechar el potencial de los sistemas de gestión integrados y todos los beneficios que ello trae para las organizaciones.

Cabe precisar que para diseñar un mecanismo que permita medir el nivel de integración de los sistemas integrados de gestión se requiere establecer un concepto único de integración y definir sus niveles; lo anterior, para garantizar un único punto de vista que pueda proporcionar un referente para compararse con la gestión que se realiza en otras organizaciones. Asimismo, este mecanismo de medición debe contar con una estructura que se ajuste a los aspectos en los cuales se integran con otros sistemas; la estructura, los conceptos y las teorías seleccionadas se configuran en una propuesta a modo de cuestionario para medir los niveles de integración que alcanzan los sistemas de gestión integrados dentro de las organizaciones. De igual modo, su viabilidad se evalúa con una validación de contenido de cada uno de los ítems que lo conforman, a través de un grupo de expertos y aplicando una prueba no probabilística, que permite medir el grado de correlación entre los expertos, con el fin de generar confianza en el instrumento para su aplicación dentro de las organizaciones.

Cuando existe más de un sistema de gestión implementado en las organizaciones se genera la necesidad de integrarlos para aprovechar en mayor 
medida sus beneficios. De acuerdo con lo afirmado por Karapetrovic y Willborn (1998), las principales ventajas y avances que se obtienen de la integración de los sistemas son la mejora del desarrollo y la transferencia tecnológica, y de la ejecución operativa; el progreso en los métodos internos de gestión y en los equipos multifuncionales, la mayor motivación del personal y el menor número de conflictos interfuncionales; la reducción y mayor coordinación de las múltiples auditorías, el aumento de la confianza de los clientes e imagen positiva en la comunidad y el mercado, la reducción de costos y una reingeniería más eficiente.

Las organizaciones que cuentan con sistemas integrados de gestión conformados por los estándares ISO 9001, ISO 14001 y OHSAS 18001 asumen un criterio particular de integración de acuerdo con el contexto donde realizan sus actividades y de la motivación que les lleve a integrarlos. Por consiguiente, actualmente no es posible generar puntos de comparación objetivos entre los niveles de integración de los sistemas de gestión de las organizaciones colombianas, puesto que en esta integración no se miden criterios estandarizados. Cabe aclarar que la propuesta presentada en este artículo está basada en las versiones ISO 9001:2008, ISO 14001:2004 y OHSAS 18001:2007.

\section{Un mecanismo para medir niveles de inteǵración}

En la actualidad existen modelos de excelencia proferidos por instituciones como la Fundación europea para la Gestión de la Calidad (EFQM, por sus siglas en inglés), con el premio Europeo a la Calidad (Moreno, 2007). La Union of Japanese Scientists and Engineers (JUSE), con el premio Deming (The W. Edwards Deming Institute, s. f.). El Instituto Nacional de Estándares y Tecnología (NIST, por sus siglas en inglés), en Estados Unidos con el Malcolm Baldrige National Quality Award, y en Colombia, la Corporación Calidad, con el Premio Nacional a la Calidad y la Innovación (2012). Estos premios son entregados a las organizaciones que se someten a una evaluación rigurosa que mide la excelencia en la gestión interna con el fin de evidenciar altos niveles de eficiencia en sus procesos, de acuerdo con ciertos criterios (ver tabla 1).

Tabla 1. Criterios en los que se basan los modelos de excelencia

\begin{tabular}{|l|l|l|l|}
\hline \multicolumn{2}{|c|}{$\begin{array}{c}\text { AGENTES } \\
\text { FACILITADORES }\end{array}$} & \multicolumn{2}{c|}{ RESULTADOS } \\
\hline \multicolumn{1}{|c|}{$\begin{array}{l}\text { Modelo } \\
\text { EFQM }\end{array}$} & \multicolumn{1}{c|}{$\begin{array}{c}\text { Modelo } \\
\text { Iberoamericano }\end{array}$} & \multicolumn{1}{c|}{$\begin{array}{c}\text { Modelo } \\
\text { EFQM }\end{array}$} & \multicolumn{1}{c|}{$\begin{array}{c}\text { Modelo } \\
\text { Iberoamericano }\end{array}$} \\
\hline $\begin{array}{l}\text { 1. } \\
\text { Liderazgo }\end{array}$ & $\begin{array}{l}\text { 1. Liderazgo } \\
\text { y estilo de } \\
\text { dirección }\end{array}$ & $\begin{array}{l}\text { 6. Resultados } \\
\text { de los clientes }\end{array}$ & $\begin{array}{l}\text { 6. Resultados } \\
\text { de los clientes }\end{array}$ \\
\hline 2. Personas & $\begin{array}{l}\text { 2. Desarrollo de } \\
\text { las personas }\end{array}$ & $\begin{array}{l}\text { 7. Resultados } \\
\text { de las } \\
\text { personas }\end{array}$ & $\begin{array}{l}\text { 7. Resultados } \\
\text { de las personas }\end{array}$ \\
\hline $\begin{array}{l}\text { 3. Política y } \\
\text { estrategia }\end{array}$ & $\begin{array}{l}\text { 3. Política y } \\
\text { estrategia }\end{array}$ & $\begin{array}{l}\text { 8. Resultados } \\
\text { en la sociedad }\end{array}$ & $\begin{array}{l}\text { 8. Resultados } \\
\text { en la sociedad }\end{array}$ \\
\hline $\begin{array}{l}\text { 4. Alianza y } \\
\text { recursos }\end{array}$ & $\begin{array}{l}\text { 4. Asociados y } \\
\text { recursos }\end{array}$ & $\begin{array}{l}\text { 9. Resultados } \\
\text { clave }\end{array}$ & $\begin{array}{l}\text { 9. Resultados } \\
\text { clave }\end{array}$ \\
\hline 5. Procesos & 5. Clientes & -- & -- \\
\hline
\end{tabular}

Fuente: Nieves y McDonnell (2006).

El aspecto más relevante para citar estos modelos de excelencia es el de cómo las instituciones detrás de los premios miden la excelencia en la gestión, específicamente los mecanismos que utilizan para ello, pues cada institución se centra en ciertos criterios y en requisitos que deben cumplir las organizaciones. Para el Modelo Deming se tiene a un comité que mide ciertos criterios en el actuar de una organización, al igual que en el Modelo Malcolm Baldrige, pero en este último se determina que se cumple con los criterios mediante una tabla de puntajes, mientras que en el modelo de EFQM y en el modelo del Premio Colombiano a la Calidad en la Gestión los criterios se 
miden de acuerdo con un cuestionario previamente definido. Tanto en el modelo Malcolm Baldrige, como en el Premio Colombiano a la Calidad en la Gestión y en el modelo EFQM los puntajes se sitúan en unos rangos previamente establecidos para cada uno de los criterios, donde al final generan una calificación total que es la que dicta si la organización cumple o no con los criterios establecidos para ganar el galardón.

Como se evidencia anteriormente por las diferentes instituciones que están detrás de los premios a la excelencia, la mejor forma de medir la gestión dentro de las organizaciones es por medio de cuestionarios previamente codificados; estos son los mecanismos que permiten generar la información necesaria para medir el desempeño en la gestión dentro de las organizaciones.

\section{Un concepto de integración enmarcado en los sistemas de gestión ISO 9001, ISO 14001 y OHSAS 18001}

La difusión de los sistemas de gestión certificables y no certificables lleva a que organizaciones donde hay implementados uno o más de estos sistemas tengan dos opciones para su gestión, que estos funcionen por separado o funcionen de manera integrada (Bernardo 2009).

A partir de la revisión de la literatura, se identificaron diversas conceptualizaciones de "integración" para los sistemas de gestión seleccionados en este estudio, los cuales se presentan en la tabla 2.

Tabla 2. Definiciones de sistema integrado de gestión

\begin{tabular}{|c|c|}
\hline AUTORES & DEFINICIONES \\
\hline $\begin{array}{l}\text { Karapetrovic y Willborn, y } \\
\text { Karapetrovic }\end{array}$ & $\begin{array}{l}\text { El conjunto de procesos interconectados que comparten un conjunto de recursos humanos, } \\
\text { información, materiales, infraestructuras y recursos financieros, a fin de lograr un conjunto de } \\
\text { objetivos relacionados con la satisfacción de una gran variedad de stakeholders. }\end{array}$ \\
\hline Labodová & $\begin{array}{l}\text { Combinación que integra la gestión de la calidad y medio ambiental con el sistema de gestión de } \\
\text { seguridad y salud. }\end{array}$ \\
\hline $\begin{array}{l}\text { Asociación Española de Normalización } \\
\text { y Certificación (AENOR) }\end{array}$ & $\begin{array}{l}\text { Conjunto formado por la estructura de la organización, las responsabilidades, los procedimientos, los } \\
\text { procesos y los recursos que se establecen para llevar a cabo la gestión integrada de los sistemas. }\end{array}$ \\
\hline Pojasek & $\begin{array}{l}\text { Combinación de sistemas de gestión utilizando un enfoque en los empleados, una visión de proceso y } \\
\text { un enfoque de sistemas, que permite poner todas las prácticas relevantes el estándar de gestión en un } \\
\text { único sistema. }\end{array}$ \\
\hline Griffith y Bhutto & $\begin{array}{l}\text { Un único sistema de gestión que reparte los procesos de la organización a través de funciones } \\
\text { específicas y con apoyo mutuo estructurado, configurados en torno a las necesidades globales de la } \\
\text { organización. }\end{array}$ \\
\hline Bernardo & $\begin{array}{l}\text { Proceso de unir diferentes sistemas de gestión en un único sistema con recursos comunes, con el } \\
\text { objetivo de mejorar la satisfacción de los stakeholders. }\end{array}$ \\
\hline MacGregor Associates & $\begin{array}{l}\text { Un único estándar de gestión básico con el soporte opcional de otros estándares que cubren } \\
\text { requerimientos específicos. }\end{array}$ \\
\hline ISO & $\begin{array}{l}\text { El proceso de unificación de los requerimientos de múltiples sistemas de gestión estandarizados en el } \\
\text { sistema de gestión global de una organización. }\end{array}$ \\
\hline
\end{tabular}

Fuente: elaboración propia, a partir de Bernardo (2009, p. 12). 
A partir de esta revisión, se asumió inicialmente para el desarrollo de la investigación el concepto propuesto por Wilkinson y Dale (1999). Estos autores mencionan que la integración de los sistemas de gestión es un proceso a través del cual los diferentes sistemas de gestión (SG) implantados en la organización se gestionan como un único sistema y no como sistemas independientes o separados; el resultado final de este proceso es un sistema integrado de gestión (SIG). Los motivos para que una organización decida realizar la integración de los SG se basan en la reducción de costos, el aprovechamiento de sinergias y el aumento de la eficiencia de la organización (Wilkinson, 1999).

Para la integración de sistemas de gestión, organizaciones como la Internacional Organization for Standardization (ISO), el Instituto Británico de Normalización (BSI) y la Asociación Española de Normalización y Certificación (AENOR) han desarrollado metodologías y estándares que tienen como propósito facilitar no solo a las organizaciones que implementan los sistemas de gestión, sino también a quienes crean los estándares que los normalizan, su posterior integración, de forma que les permita identificar los puntos clave dentro de los mismos sistemas de gestión y a su saber vez cuáles son las condiciones dentro de las organizaciones para lograrlo.

En 2006, el BSI creó la especificación disponible al público Publicly Available Specification (PAS 99: 2012). Esta es una especificación de requisitos comunes del sistema de gestión como marco para la integración y se desarrolla como respuesta a la demanda del mercado de alinear los procesos y procedimientos en una estructura holística que permita a las organizaciones operar con mayor eficacia. La implementación de este estándar intenta simplificar la implantación de múltiples sistemas de gestión en un único sistema de gestión global dentro de las organizaciones, por medio de la consolidación de los requisitos comunes de los sistemas de gestión.

A nivel administrativo o de entidades certificadoras, se han publicado diversas normas a nivel nacional e internacional para ello. Diferentes países han desarrollado estándares o guías para la integración de sistemas, como es en España el estándar AENOR-UNE 66177 en su versión 2005, el cual es una guía para la integración de los sistemas de gestión dentro de todo tipo de organizaciones; es una norma que no pretende reemplazar las pautas existentes de sistemas de gestión, sino que

[...] proporciona directrices para desarrollar, implantar y evaluar el proceso de integración de los sistemas de gestión de la calidad, ambiental y seguridad y salud en el trabajo de aquellas organizaciones que han decidido integrar total o parcialmente dichos sistemas en busca de una mayor eficacia en su gestión y de aumentar su rentabilidad. (Asociación Española de Normalización y Certificación [AENOR], 2005, p. 4)

Asimismo indica que el proceso de integración tiene como finalidad "la definición e implantación en condiciones controladas de un plan de integración desarrollado específicamente en función de los objetivos, contexto y nivel de madurez de la organización" (Bucci, 2011, p. 10). Apoyado en la metodología PHVA (Planear, Hacer, Verificar y Actuar), al igual que ISO 14001 y OHSAS 18001, muestra cuál debe ser la estructura para la integración, cómo debe ser el desarrollo del plan de integración, cómo se debe realizar la implantación del plan de integración y cómo hacer la revisión y mejora del sistema integrado de gestión (Bucci, 2011).

Por su lado, la ISO ha publicado una metodología para el uso integrado de normas de sistemas de gestión en un libro publicado en 2008 con el mismo nombre en inglés The Integrated Use of Management System Standards 
(Internacional Organization for Standardization, 2008), en el que se proporcionan ejemplos, descubrimientos, retos y beneficios para aquellas organizaciones que consideran la implantación de los requerimientos de múltiples sistemas de gestión a través de un enfoque integrado. Esta metodología sigue siete pasos para lograr la integración: 1) promover la integración; 2) determinación del ámbito o alcance de la integración; 3) planificación de la integración; 4) conectar los requerimientos de los sistemas de gestión y el sistema de gestión de la organización; 5) incorporar los requerimientos de los sistemas de gestión estandarizados en el sistema de gestión de la organización; 6) mantener y mejorar la integración, y 7) aplicar las lecciones aprendidas en la organización.

\section{Estrategias y metodologías para la integración de sistemas de gestión}

Las estrategias para la integración varían dependiendo del orden como se implementen los sistemas de gestión que se desean integrar. Para lograr esto existen varias estrategias, una de ellas es la propuesta por Karapetrovic y Willborn (1998), que se ha encontrado como la más conocida y citada para lograrlo. Esta estrategia se basa en dos etapas; la primera está sustentada en el sistema de gestión de la calidad y el sistema de gestión ambiental, y en determinar el orden con el que se implementan para la organización, si primero el de la calidad y segundo el ambiental o viceversa; de igual forma, si se implementaran de manera simultánea. En la segunda etapa, se parte del hecho de si la empresa cuenta, además del sistema de gestión de la calidad y el sistema de gestión ambiental, con otros sistemas de gestión implementados (Jonker y Karapetrovic, 2003).

Además de los estándares creados alrededor del tema de la integración de sistemas de gestión dentro de las organizaciones, algunos autores reconocidos también han construido varias metodologías para lograrlo, como
Karapetrovic, Willborn, Aboulnaga, Weiler, Renfrew y Muir, Puri, entre otros. Una de las metodologías más destacada y coherente con las estrategias de integración para los sistemas de gestión anteriormente vistas es la propuesta realizada por Karapetrovic y Willborn (1998), la cual tiene como punto de partida unos objetivos previamente establecidos por la organización en su intención de integrar los sistemas de gestión.

Jonker y Karapetrovic (2003) proponen el enfoque por procesos para la integración de los sistemas de gestión, el cual indican que es útil para todas las organizaciones y tiene como eje central los procesos de auditoría, partiendo del que "los sistemas a integrar tienen estructuras y conceptos similares que los hacen integrables" (Bernardo, 2009, p. 116). Esta metodología implicaba la unificación de los objetivos, procesos y recursos de las auditorías en los sistemas de gestión de la calidad, ambiental, seguridad y salud en el trabajo y otros sistemas de gestión, lo que daba como resultado informes y acciones preventivas y correctivas integradas, así como las oportunidades de mejora para el sistema integrado.

Posteriormente, en 2004 propusieron otro modelo que comenzaba por integrar los requerimientos comunes de los sistemas de gestión en un elemento central que se convertiría en el núcleo del sistema integrado de gestión; así, los requerimientos específicos permanecen en módulos funcionales. De esta manera, las diferencias entre cada estándar son fáciles de reconocer, después de ello, los requerimientos inherentes a un estándar se utilizan como denominador común para un requerimiento del sistema integrado de gestión y no hay la necesidad de separarlos en módulos funcionales. Con esto se lograría alinear los sistemas para dar lugar a su integración (Jonker y Karapetrovik, 2004).

Otro de los modelos importante por mencionar es el de integración basado en sinergias de Zeng. Dentro de este se propone un modelo "sinergético" para 
implantar un sistema integrado de gestión en tres diferentes niveles (Zeng y Shi, 2007): en un primer nivel se da la sinergia estratégica (objetivos, planes y acciones estratégicos de la calidad, medioambiente y seguridad y salud); en el segundo nivel, la sinergia de los recursos (recursos humanos, financieros), estructural (compromiso desde la alta dirección para la integración y su mantenimiento) y cultural (cultura organizacional para la integración y la mejora continua), y en un tercer nivel, la sinergia de la documentación (toda la documentación).

Castillo y Martínez (2010) proponen un enfoque para combinar e integrar la gestión de sistemas, donde desde el enfoque por procesos y la mejora continua con el ciclo de Deming (PHVA) se plantea una guía para la integración, partiendo de un esquema de combinación de sistemas sin integración y se expone un enfoque práctico para la combinación integrada de estos analizado desde la perspectiva de conceptos como innovación, competitividad, cumplimiento legal corporativo y la aceptación social y gubernamental.

\section{Niveles de integración enmarcado en los sistemas de gestión para ISO 9001, ISO 14001 y OHSAS 18001}

Cuando una organización decide integrar sus sistemas de gestión, ya sea utilizando las guías de los organismos de normalización o las teorías propuestas por algún teórico, para ir más allá y aprovechar los beneficios que trae consigo un sistema integrado de gestión debe tener en cuenta otro aspecto, como el nivel de integración, el cual se verá reflejado dependiendo de lo que se quiera alcanzar dentro de la misma organización (Bernardo, 2009). En la tabla 3 se resumen algunos niveles de integración para sistemas de gestión expuestos por algunos autores.

En todas estas escalas de niveles, algunos consideran un primer nivel de no integración donde los sistemas operan por separado; en otros casos, se considera un nivel inicial donde hay una mínima o nula integración. Asimismo, todos los teóricos consideran niveles intermedios, en los cuales la integración se torna en una etapa de transición entre la no integración y la total integración.

Tabla 3. Niveles de integración propuestos por varios autores

\begin{tabular}{|l|l|}
\hline \multicolumn{1}{|c|}{ AUTOR } & \multicolumn{1}{c|}{ NIVELES PROPUESTOS } \\
\hline \multirow{5}{*}{ Seghezzi (1997) } & $\begin{array}{l}\text { 1. Adición: los sistemas parciales para calidad, medio ambiente, etc. están separados y descritos en } \\
\text { documentos separados, pero el contenido se hace comparable. }\end{array}$ \\
\cline { 2 - 3 } & $\begin{array}{l}\text { 2. Fusión: las instrucciones de trabajo están completamente integradas, pero no los procedimientos ni el } \\
\text { manual; se crea un sistema total, pero el sistema parcial o subsistemas todavía son visibles. }\end{array}$ \\
\cline { 2 - 3 } Wilkinson y y Dale (1999) & $\begin{array}{l}\text { 3. Integración: las empresas pueden elegir o desarrollar un SG genérico como su sistema general e incluir } \\
\text { todos los sistemas parciales. }\end{array}$ \\
\hline & $\begin{array}{l}\text { 1. El primer nivel: se refiere a SGS individuales, en el que el sistema es integrado en cada una de las } \\
\text { funciones y actividades de la organización. }\end{array}$ \\
\cline { 2 - 3 } & $\begin{array}{l}\text { 3. El tercer nivel: implica integrar ciertas partes de los SGS con otros sistemas certificados, pero sin utilizar los combinación de sistemas basados en los enlaces identificados entre los SGS. La } \\
\text { enlaces identificados. }\end{array}$ \\
\hline
\end{tabular}




\begin{tabular}{|c|c|}
\hline AUTOR & NIVELES PROPUESTOS \\
\hline Wilkinson y y Dale (1999) & $\begin{array}{l}\text { 4. El cuarto nivel: consiste en integrar los sistemas certificados y no certificados en un SG total, con las } \\
\text { políticas y objetivos alineados con y apoyando la estrategia, la política y los objetivos globales de la empresa. }\end{array}$ \\
\hline \multirow{3}{*}{ Kirkby (2002) } & $\begin{array}{l}\text { 1. El primer nivel - separado: donde los SGS cubren sus áreas diferenciadas para cada conjunto de } \\
\text { requerimientos. Los estándares cubren áreas separadas de gestión y permite a la organización decidir si opera } \\
\text { separadamente. Comercialmente tiene la desventaja de crear duplicación de esfuerzo. }\end{array}$ \\
\hline & $\begin{array}{l}\text { 2. En el segundo nivel - alineado: los SGS hacen uso de las áreas comunes de los estándares, como } \\
\text { las auditorías internas, la revisión del sistema, las acciones correctivas, la formación, la organización y } \\
\text { responsabilidad, y la política. Este hecho permite alinear todos los elementos comunes como la revisión del } \\
\text { sistema y las auditorías internas, que se distribuyen hacia el mismo sistema, y mantener por separado los } \\
\text { subsistemas específicos. }\end{array}$ \\
\hline & $\begin{array}{l}\text { 3. El tercer nivel - integrado: combina todos los estándares en un sistema de gestión común). Algunas de las } \\
\text { ventajas son reducción del riesgo, de la duplicación, añade valor y ventaja competitiva a la empresa, aumenta la } \\
\text { coordinación entre departamentos, etc. }\end{array}$ \\
\hline \multirow{3}{*}{ Karapetrovic (2002) } & $\begin{array}{l}\text { 1. Aquellas organizaciones que han inteǵrado solo la documentación, creando un manual común y } \\
\text { procedimientos específicos, que pueden mantenerse separados o integrados completamente. Esta integración } \\
\text { total también se ha de lograr para las instrucciones de trabajo y registros. }\end{array}$ \\
\hline & $\begin{array}{l}\text { 2. Aquellas organizaciones que han alineado los procesos, objetivos y recursos, integra la planificación, } \\
\text { diseño, implantación y otras actividades. }\end{array}$ \\
\hline & $\begin{array}{l}\text { 3. Aquellas que han integrado todas las partes del sistema de gestión en un único sistema de gestión } \\
\text { (all-in-one system). }\end{array}$ \\
\hline \multirow{2}{*}{ Karapetrovic (2003) } & $\begin{array}{l}\text { 1. Integración parcial: puede graduar desde la simple colaboración hasta la alineación y armonización de los } \\
\text { objetivos, procesos y recursos de sistemas de gestión separados. }\end{array}$ \\
\hline & $\begin{array}{l}\text { 2. Integración total: los SGS constituyentes pierden sus identidades únicas, y resultan en una completa } \\
\text { integración en un único sistema integrado de gestión. }\end{array}$ \\
\hline \multirow{3}{*}{$\begin{array}{l}\text { Beckmerhagen et al } \\
\text { (2003) }\end{array}$} & 1. Armonización: las organizaciones han integrado la documentación a un nivel parcial. \\
\hline & 2. Cooperación: denota la mejora de los sistemas combinados utilizando auditorías y recursos integrados. \\
\hline & 3. Amalgamación: se logra la integración total de los SGS en un SIG nuevo y global. \\
\hline \multirow{4}{*}{ Pojasek (2006) } & $\begin{array}{l}\text { 1. Combinado: significa que los SGS específicos están siendo utilizados al mismo tiempo en la misma } \\
\text { organización. }\end{array}$ \\
\hline & 2. Integrable: se refiere a la identificación de elementos comunes. \\
\hline & 3. Integrante: denota la integración de estos elementos comunes. \\
\hline & 4. Integrado: significa que se logra un sistema que incorpora todos los elementos comunes. \\
\hline \multirow{3}{*}{ Jørgensen (2008) } & 1. Correspondencia: referencias cruzadas y coordinación interna. \\
\hline & 2. Genérico: consiste en entender los procesos y las tareas genéricos en el ciclo de gestión. \\
\hline & $\begin{array}{l}\text { 3. Integración: creación de una cultura de aprendizaje, participación de los stakeholders y mejora continua de } \\
\text { la performance. }\end{array}$ \\
\hline
\end{tabular}

Fuente: elaboración propia, a partir de Bernardo (2009). 


\section{METODOLOGÍA}

Un estudio para definir un mecanismo que permita medir niveles de integración en sistemas integrados de gestión se define como exploratorio, esto explicado desde la perspectiva de que no existe algo similar en la actualidad; asimismo, tiene un enfoque cualitativo, ya que se exploró un fenómeno en profundidad a partir de una teoría adoptada. Para el caso de este estudio fue el concepto de integración y de una teoría de niveles de integración, de los cuales se realizará una revisión bibliográfica y se seleccionará el concepto y la teoría más propicia. A partir de ello se desarrolló la propuesta del instrumento que permite medir el nivel de integración de los sistemas de gestión. Este enfoque se basa en explorar y describir con el fin de generar perspectivas teóricas, que van de lo particular a lo general (Hernández, Fernández y Baptista, 2010, p. 51).

Un instrumento que se crea para medir niveles de integración se debe enmarcar en un concepto único de integración y una teoría seleccionada sobre niveles de integración, que se articulan con los requisitos comunes de las normas ISO 9001, ISO 14001 y OHSAS 18001 para su construcción.

Después de identificar los aspectos teóricos en los cuales se basó el instrumento, se continuó con la construcción del instrumento articulando los tres sistemas de gestión objeto de la evaluación, en una serie de ítems distribuidos de acuerdo con los criterios que abarca cada nivel de integración. Posteriormente, se realizó una validación de su contenido para verificar la claridad, pertinencia y suficiencia de cada uno de los ítems propuestos; por último, se aplicó una prueba piloto en una empresa que cuenta con los tres sistemas de gestión para verificar en un ambiente real si los resultados de la aplicación suministran la información que se plantea de forma teórica en la construcción del instrumento (ver figura 1).
Figura 1. Proceso de estructuración del instrumento

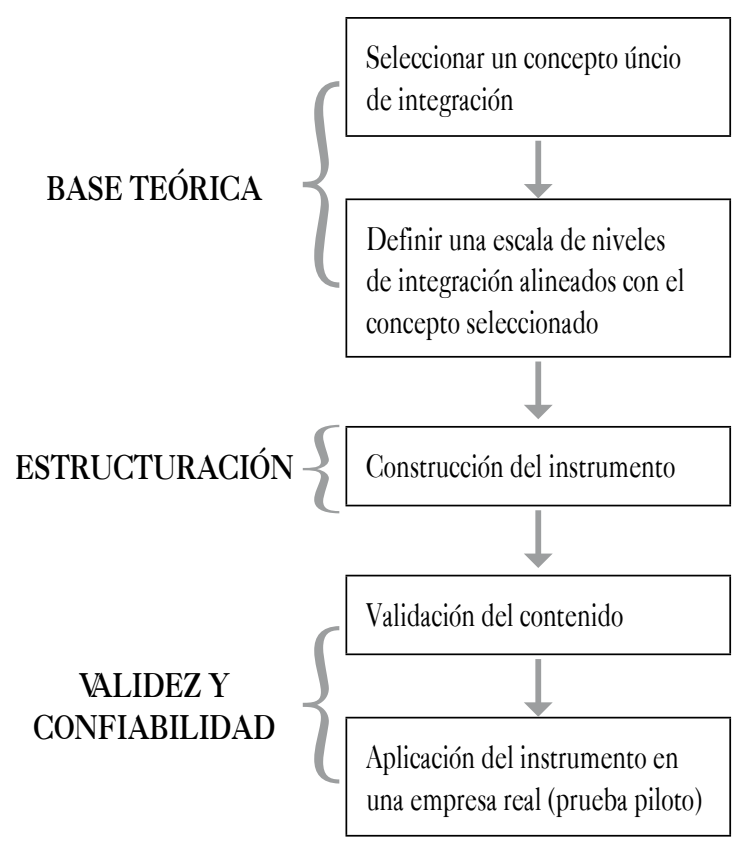

Fuente: elaboración propia.

Cabe destacar que el instrumento fue construido con base en los hallazgos de la revisión de la literatura presentada y las normas PAS 99 (British Standar Institution, 2008, p. 26) y UNE 66177.

La validación del instrumento de medición se realizó considerando la validez de contenido, con el fin de determinar si el instrumento mide las principales dimensiones de las variables propuestas. Para ello un grupo de expertos en sistemas integrados de gestión, con experiencia superior a 10 años y con estudios de postgrados relacionados con sistema de gestión de calidad, ambiental y seguridad y salud, sometieron a evaluación la herramienta para sus 52 ítems propuestos inicialmente, y así se determinó si el contenido es suficiente, pertinente y claro. Adicionalmente, se aplicó una prueba no probabilista (coeficiente de Kendall) con el fin de medir el grado de correlación entre los expertos. Como resultado de lo anterior se realizaron los ajustes al instrumento sugeridos por los expertos y se codificó el instrumento con ayuda de estadistas; 
esto para facilitar el análisis de la información después de aplicar el instrumento en una organización. Por último, se sometió a una prueba piloto aplicándolo en una empresa que cuenta con más de tres años con sistemas de gestión certificados en ISO 9001, ISO 14001 y OHSAS 18001, con el fin de observar la viabilidad de su aplicación.

\section{RESULTADOS Y DISCUSIÓN}

\section{Mecanismo para medir la integración de sistemas de gestión}

Los criterios y valores en los que están basados los modelos de excelencia son referentes de una gestión y unos resultados sobresalientes de las organizaciones que son reconocidas como "excelentes". Es por ello la rigurosidad que se aplica por parte de las instituciones para determinar si una organización está asumiendo los criterios establecidos por el modelo y cuenta con los valores que requiere la excelencia en la gestión. En este proceso se evalúan diferentes aspectos en los que dependiendo de cada modelo se profundizan, se va sumando un puntaje de acuerdo con lo encontrado y al final se llega a un puntaje que, como resultado de lo estipulado en la evaluación del modelo otorgará o no el premio a la excelencia en la gestión.

Por lo anterior, esta es la forma que se toma como referencia para el mecanismo que se plantea en este trabajo para medir niveles de integración de sistemas integrados de gestión, ya que se requiere una alta rigurosidad que permita determinar los aspectos claves en los que se integran los sistemas, teniendo como referentes un concepto único de integración y unos niveles de integración determinados, donde la encuesta construida alrededor de estos criterios será el instrumento utilizado para tal fin.

\section{Un concepto genérico de integración}

Para el propósito del instrumento es determinante tener una posición lo más general posible en cuanto a lo que significa la integración de sistemas de gestión. Con la intención de abarcar el concepto más amplio de esta definición, se tuvieron en cuenta los puntos claves en los que cada norma y autor convergen para lograr la integración de sistemas de gestión.

En la revisión de los documentos creados por los organismos internacionales de normalización se puede concluir que el concepto de integración va dirigido a integrar todo lo común en un único sistema de gestión y posteriormente vincular a este los aspectos inherentes de cada sistema.

De acuerdo con lo que se revisó de los teóricos y lo que es la integración para los organismos internacionales de normalización, como marco del instrumento de medición de niveles de integración, se hace referencia al concepto de sistema integrado de gestión, referenciado por Casadesus, Heras y karapetrovic (2009) como "[...] el proceso de unir diferentes sistemas de gestión en un único sistema con recursos comunes con el objetivo de mejorar la satisfacción de las partes interesadas" (p. 743).

La anterior definición se refiere a que al obtenerse un único sistema global de gestión dentro de las organizaciones se consigue mayores beneficios que se traducen a su vez en mayores niveles de satisfacción de los stakeholders. Este enfoque logra una visión más holística de la organización, ya que otras definiciones solo enmarcan el concepto de sistema integrado como unificación de requisitos, un solo sistema de gestión como base para integrar otros sistemas, o un enfoque solo por procesos o funciones. Estas definiciones por separado no son suficientes para el propósito de esta investigación; además, no permiten alcanzar plenamente los 
beneficios que un sistema integrado de gestión puede brindar a las organizaciones.

\section{Una escala de niveles para medir la integración de sistemas}

El objetivo principal del instrumento de medición de niveles de integración es la categorización del nivel de integración en el que se encuentran los sistemas de gestión dentro de una organización.; para ello, es importante definir estos niveles y que estén alineados al concepto de integración anteriormente seleccionado. Al revisar los documentos normativos creados por los organismos internacionales de estandarización, no se encontró información específica acerca de niveles de integración de sistemas de gestión integrados. Es por esto que para el propósito del instrumento, y alineado a la definición de sistema integrado de gestión seleccionada, la teoría de niveles para sistemas integrados de gestión que mejor se adapta es la de los tres niveles de integración propuestos por Karapetrovic (2002), ya que en ellos se logran abarcar todos los aspectos que son parte de la integración de sistemas de gestión que se indican en los documentos de los organismos internacionales de estandarización referenciados y en los que concuerdan la mayoría de los demás autores consultados, empezando en un primer nivel por la integración de la documentación; en un segundo nivel, alineando los procesos, objetivos y recursos, y en un tercer nivel integrando todas las partes del sistema de gestión en un único sistema. Estos niveles permiten enmarcar la evolución de la integración de los sistemas y proporcionan puntos claves que permiten fijar un rumbo claro para alcanzar los objetivos de la integración de sistemas de gestión (ver tabla 4).

Tabla 4. Niveles de integración propuestos por Karapetrovic

\begin{tabular}{|l|l|}
\hline \multicolumn{1}{|c|}{ NIVELES DE INTEGRACIÓN } & \multicolumn{1}{c|}{ DESCRIPCIÓN } \\
\hline Integrar la documentación & $\begin{array}{l}\text { Crear un manual específico de calidad, del medio ambiente, y otros procedimientos requeridos. } \\
\text { Un ejemplo de una integración completa sería un procedimiento único para la planificación } \\
\text { de la gestión que se utiliza para la calidad, el medio ambiente, la seguridad, la confiabilidad o } \\
\text { cualquier otro aspecto del rendimiento. La integración completa también debe lograrse para las } \\
\text { instrucciones de los trabajos y registros; sin embargo, pueden necesitar ser mantenidos algunos } \\
\text { registros de función específica. }\end{array}$ \\
\hline $\begin{array}{l}\text { Alinear los procesos básicos, objetivos } \\
\text { y recursos }\end{array}$ & $\begin{array}{l}\text { Racionalizar el uso de los procesos centrales por integración de la planificación, diseño, } \\
\text { implementación y otras actividades verticales a través de los sistemas de gestión. Vincular } \\
\text { funciones específicas y objetivos, y el uso de los recursos humanos, financieros y materiales. } \\
\text { Para este paso se espera conseguir mayores beneficios en términos de ahorro de recursos y } \\
\text { proporcionar así efectos de sinergia. }\end{array}$ \\
\hline Crear un sistema "todo en uno" & $\begin{array}{l}\text { Este sería el último paso en cualquier esfuerzo de integración. Debido a las similitudes } \\
\text { conceptuales importantes entre los sistemas actuales, un sistema de gestión "universal" para } \\
\text { la calidad, la fiabilidad, el medio ambiente, la salud ocupacional y la seguridad, e incluso la } \\
\text { responsabilidad social no es un objetivo descabellado. Los sistemas de calidad, ambiental y } \\
\text { seguridad, y salud pierden su identidad propia dentro de la organización y se fusionan en el } \\
\text { marco común del sistema de gestión de la organización. }\end{array}$ \\
\hline
\end{tabular}

Fuente: elaboración propia, a partir de Karapetrovic (2002, p. 6). 


\section{Integración de la documentación, alinear los procesos básicos, objetivos y recursos}

La estructuración del instrumento se planteó a partir de los requisitos comunes de cada sistema de gestión objeto de integración. Para la integrar la documentación, se abordaron los requisitos de cada norma que se refiere a la documentación del sistema de acuerdo con los niveles propuestos; asimismo, para la alineación de los procesos básicos, objetivos y recursos, que representa el segundo nivel de integración. Estos se relacionan con los requisitos que son comunes para los tres sistemas de gestión ISO 9001, ISO 14001 y OHSAS 18001. Dicha conexión dio como resultado unas dimensiones. De lo anterior se plantearon los cuestionamientos a modo de afirmaciones para cada dimensión, para así indagar acerca de la integración de estos aspectos dentro de las organizaciones.

Para los ítems desarrollados en el Nivel I. Documentación y Nivel II. Procesos básicos, objetivos y recursos, las opciones de respuesta se plantean de modo que se relacionen directamente con los sistemas de gestión que se integran para cada dimensión. Para ello se establecieron las siguientes opciones: 1) todos los sistemas de gestión; 2) los sistemas de gestión de la calidad y ambiental; 3) los sistemas de gestión de calidad y seguridad y salud; 4) los sistemas de gestión ambiental y seguridad y salud, y 5) ninguno de los sistemas de gestión.

Cada opción indica cuáles sistemas de gestión integran la organización de acuerdo con el propósito con el cual es planteado cada ítem. Como se observa, se establecen todas las combinaciones posibles para los tres sistemas de gestión y una más para cuando no se integra ninguno de ellos.
La opción 1 indica que la organización para la dimensión evaluada integra los tres sistemas de gestión, es decir, hay una integración total en esa dimensión, por eso esta opción representará el puntaje más alto para la evaluación; la opción 5 indica que la organización no integra para la dimensión evaluada ninguno de los sistemas de gestión; en otras palabras, que cada uno funciona separadamente, por lo tanto se le otorga el puntaje más bajo al no existir integración. Para las opciones 2, 3 y 4, que realizan combinaciones entre dos sistemas de gestión que funcionan integradamente, se otorga un puntaje intermedio que indica una integración parcial de los sistemas. Los valores para cada una de estas respuestas se determinarán más adelante cuando se realice la validez del instrumento construido.

\section{Crear un sistema de gestión "todo en uno"}

Establecer un sistema de gestión global para la organización representa el último nivel de integración (Nivel III), donde cada sistema de gestión estandarizado por ISO 9001, ISO 14001 y OHSAS 18001 pierde su identidad y se fusiona en el marco común del sistema de gestión de la organización. Teniendo en cuenta lo anterior, se configuran ítems para el instrumento de acuerdo con las siguientes dimensiones: nivel gerencial, compromiso de la dirección, eficacia del sistema, comunicación, nivel táctico, nivel operacional e identidad de los sistemas de gestión.

Estas dimensiones se dirigen a los aspectos que se refieren a indagar acerca de la total integración de los sistemas de gestión en cuestión dentro de las organizaciones; de allí se plantean los cuestionamientos a modo de afirmaciones para medir el grado de percepción de integración de los sistemas dentro de la organización. 


\section{Dimensiones y codificación del instrumento de medición}

La codificación permitirá realizar un análisis rápido de la información obtenida después de la aplicación del instrumento.

Para definir los índices fue necesario darle un peso numérico a cada una de las opciones de respuesta para que se pueda estimar un puntaje máximo y uno mínimo; este se determina como se muestra en la tabla 5.

Tabla 5. Peso establecido para las opciones de respuesta Niveles I y II

\begin{tabular}{|l|c|}
\hline \multicolumn{1}{|c|}{ RESPUESTA } & VALOR \\
\hline Todos los sistemas de gestión. & 5 \\
\hline $\begin{array}{l}\text { Los sistemas de gestión de la calidad y } \\
\text { ambiental. }\end{array}$ & 3 \\
\hline $\begin{array}{l}\text { Los sistemas de gestión de calidad y } \\
\text { seguridad y salud. }\end{array}$ & 3 \\
\hline $\begin{array}{l}\text { Los sistemas de gestión ambiental y } \\
\text { seguridad y salud. }\end{array}$ & 1 \\
\hline $\begin{array}{l}\text { Ninguno de los sistemas de gestión o están } \\
\text { por separado. }\end{array}$ & 3 \\
\hline
\end{tabular}

Fuente: elaboración propia.

Como se puede observar en la tabla 5, se otorga el mayor valor numérico a la opción "Todos los sistemas de gestión", lo que quiere decir que el sistema está integrado para esa variable específica; seguido, se encuentra la misma calificación para las tres respuestas siguientes, debido a que dentro de esta investigación se considera que estar integrado el sistema de gestión de calidad con ambiental no debe tener una calificación diferente que si se encuentra integrado el sistema de gestión de calidad con el de seguridad y salud, ya que no fue objeto de esta investigación definir cuál sistema de gestión es más compatible con otro; la última opción de respuesta "Ninguno de los sistemas de gestión o están por separado" tiene el menor valor.

Para los ítems del Nivel III, se escoge una escala de Likert, la cual se dispone en cinco opciones de respuesta (ver tabla 6).

Tabla 6. Peso establecido para las opciones de respuesta - Nivel III

\begin{tabular}{|c|l|c|}
\hline RESPUESTA & \multicolumn{1}{|c|}{ DESCRIPCIÓN } & VALOR \\
\hline 1 & Totalmente de acuerdo. & 5 \\
\hline 2 & De acuerdo. & 4 \\
\hline 3 & Ni de acuerdo/ni en desacuerdo. & 3 \\
\hline 4 & En desacuerdo. & 2 \\
\hline 5 & Totalmente en desacuerdo. & 1 \\
\hline
\end{tabular}

Fuente: elaboración propia.

Como se observa en la tabla 6, se da un mayor puntaje a la opción 1, porque la forma como están dispuestos los cuestionamientos para este nivel permite que esta opción sea la que percibe un mayor nivel de integración, y las siguientes opciones de respuesta como percepciones de conformidad que se disponen en menor medida hasta una nula conformidad en la opción 5.

De acuerdo con el número de preguntas resultantes para cada nivel de integración se obtuvo un puntaje mínimo y uno máximo de cada nivel (ver tabla 7).

Tabla 7. Rango de puntajes a alcanzar en los niveles I, II y III

\begin{tabular}{|c|c|c|c|}
\hline NIVELES & $\begin{array}{c}\text { NO } \\
\text { PREGUNTAS }\end{array}$ & $\begin{array}{c}\text { PUNTAJE } \\
\text { MÁXIMO }\end{array}$ & $\begin{array}{c}\text { PUNTAJE } \\
\text { MÍNIMO }\end{array}$ \\
\hline I & 7 & 35 & 7 \\
\hline II & 21 & 105 & 21 \\
\hline III & 9 & 45 & 9 \\
\hline
\end{tabular}

Fuente: elaboración propia. 
Posteriormente, se ponderan los resultados teniendo en cuenta el peso propuesto para cada nivel, con el fin de determinar los valores máximos y mínimos que se pueden obtener en el instrumento.

El peso para cada nivel se definió en relación con el número de preguntas que tiene asociada por nivel.

$$
\begin{gathered}
\text { Máx }=35^{*} 0,19+105^{*} 0,57+45^{*} 0,24 \\
\text { Máx }=77,3 \\
\text { Min }=7 * 0,19+21 * 0,57+9 * 0,24 \\
\text { Min }=15,46
\end{gathered}
$$

La diferencia de los rangos máximo y mínimo (77,3$15,46)$ es de 61,84 , el cual es divido en 3 para obtener los índices y la amplitud de los tres niveles, lo que arroja un valor de 20,6.

Por lo tanto, los rangos para cada uno de los niveles son los que se muestra en la tabla 8 .

Tabla 8. Rango de puntajes ponderados a alcanzar en los niveles I, II y III*

\begin{tabular}{|l|c|c|}
\hline \multicolumn{1}{|c|}{ NIVELES } & $\begin{array}{c}\text { RANGO } \\
\text { MÁXIMO }\end{array}$ & $\begin{array}{c}\text { RANGO } \\
\text { MÍNIMO }\end{array}$ \\
\hline $\begin{array}{l}\text { Nivel III (Un sistema de gestión } \\
\text { "todo en uno"). }\end{array}$ & 77,3 & 56,69 \\
\hline $\begin{array}{l}\text { Nivel II (Alineación de los } \\
\text { procesos básicos, objetivos y } \\
\text { recursos). }\end{array}$ & 56,69 & 36,07 \\
\hline $\begin{array}{l}\text { Nivel I (Documentación } \\
\text { integrada). }\end{array}$ & 36,07 & 15,46 \\
\hline $\begin{array}{l}\text { *Por ejemplo, al 15,46 (mínimo) se le suma 20,6 y da 36,07 a 36,07 } \\
\text { se le suma 20,6 y da 56,69 y, a 56,69 se le suma 20,6 y da 77,3 } \\
\text { (máximo) }\end{array}$ \\
\hline
\end{tabular}

Fuente: elaboración propia.
La codificación realizada anteriormente permite determinar después de la aplicación del instrumento rápidamente el nivel de integración donde se encuentran los sistemas de gestión de la organización, pero para realizar un análisis más profundo de la información obtenida se debe realizar uno cualitativo de acuerdo con los resultados conseguidos en las respuestas recibidas; esto, debido a la situación particular de cada organización en cuanto al escenario donde están configurados sus sistemas de gestión, pero sin salirse del marco dentro del cual está construido el instrumento aquí propuesto.

Este análisis, desde el propósito que tiene la herramienta de medición, debe realizarse teniendo en cuenta, dentro de lo estipulado para cada nivel de integración, las dimensiones establecidas y las respuestas que se seleccionan para cada ítem. Un ejemplo de ello se puede observar en el anexo 7, en el que se realiza inicialmente un análisis de los datos numéricos obtenidos y después uno más profundo a partir de las respuestas recibidas.

En la tabla 9 se presenta la versión final del instrumento diseñado para medir el nivel de integración de los sistemas de gestión en organizaciones colombianas certificadas en ISO 9001:2008, ISO 14001:2004 y OHSAS 18001:2007. 
Tabla 9. Instrumento para medir el nivel de integración de los sistemas de gestión

\begin{tabular}{|c|c|c|c|c|c|c|c|}
\hline \multicolumn{8}{|c|}{ NIVEL I } \\
\hline \multirow[b]{3}{*}{ DIMENSIONES } & \multirow[b]{3}{*}{ ITEM } & \multirow[b]{3}{*}{ ÍTEM } & \multicolumn{5}{|c|}{ ESCALA } \\
\hline & & & A & B & $\mathrm{C}$ & $\mathrm{D}$ & $\mathrm{E}$ \\
\hline & & & 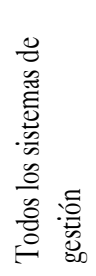 & 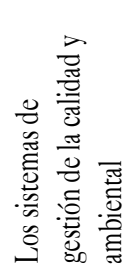 & 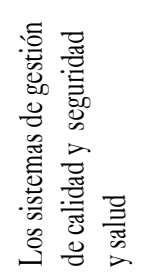 & 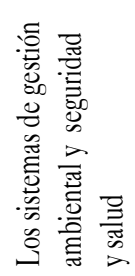 & 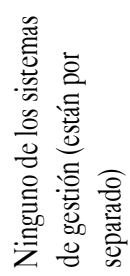 \\
\hline Manual & 1 & $\begin{array}{l}\text { En el manual } \\
\text { de gestión están } \\
\text { integrados: }\end{array}$ & 5 & 3 & 3 & 3 & 1 \\
\hline Políticas y objetivos & 2 & $\begin{array}{l}\text { Las directrices } \\
\text { estratégicas están } \\
\text { documentadas en } \\
\text { forma integrada } \\
\text { para: }\end{array}$ & 5 & 3 & 3 & 3 & 1 \\
\hline $\begin{array}{l}\text { Procedimiento } \\
\text { control de } \\
\text { documentos }\end{array}$ & 3 & $\begin{array}{l}\text { Los } \\
\text { procedimientos } \\
\text { documentados }\end{array}$ & 5 & 3 & 3 & 3 & 1 \\
\hline $\begin{array}{l}\text { Procedimiento } \\
\text { control de registros }\end{array}$ & & $\begin{array}{l}\text { los documentos y } \\
\text { registros integran: }\end{array}$ & & & & & \\
\hline $\begin{array}{l}\text { Procedimiento de } \\
\text { auditorias }\end{array}$ & 4 & $\begin{array}{l}\text { La información } \\
\text { documentada para } \\
\text { llevar a cabo las } \\
\text { auditorías internas } \\
\text { incluye: }\end{array}$ & 5 & 3 & 3 & 3 & 1 \\
\hline $\begin{array}{l}\text { Procedimiento de } \\
\text { acciones correctivas y } \\
\text { acciones preventivas }\end{array}$ & 5 & $\begin{array}{l}\text { El documento } \\
\text { para la toma } \\
\text { de acciones } \\
\text { correctivas y } \\
\text { preventivas } \\
\text { integra: }\end{array}$ & 5 & 3 & 3 & 3 & 1 \\
\hline $\begin{array}{l}\text { Instructivos de } \\
\text { trabajo integrados }\end{array}$ & 6 & $\begin{array}{l}\text { Existe información } \\
\text { documentada para } \\
\text { instrucciones de } \\
\text { trabajo donde se } \\
\text { integren: }\end{array}$ & 5 & 3 & 3 & 3 & 1 \\
\hline Registros integrados & 7 & $\begin{array}{l}\text { Se registra para los } \\
\text { controles comunes } \\
\text { información que } \\
\text { incluye: }\end{array}$ & 5 & 3 & 3 & 3 & 1 \\
\hline
\end{tabular}




\begin{tabular}{|c|c|c|c|c|c|c|c|}
\hline \multicolumn{8}{|c|}{ NIVEL II } \\
\hline \multirow[b]{3}{*}{ DIMENSIONES } & \multirow[b]{3}{*}{$\begin{array}{c}\text { NUEVO } \\
\text { CONTENIDO }\end{array}$} & \multirow[b]{3}{*}{ PREGUNTA } & \multicolumn{5}{|c|}{ ESCALA } \\
\hline & & & A & B & $\mathrm{C}$ & $\mathrm{D}$ & $\mathrm{E}$ \\
\hline & & & 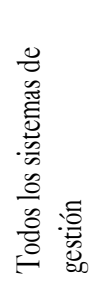 & 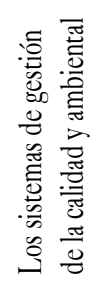 & 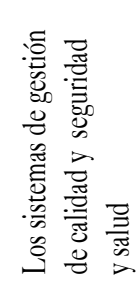 & 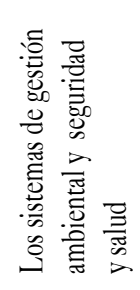 & 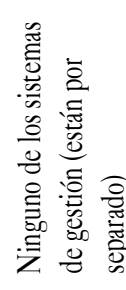 \\
\hline Visión por procesos & 8 & $\begin{array}{l}\text { Se tiene un enfoque por } \\
\text { procesos dentro de la } \\
\text { organizaciones donde se } \\
\text { integran: }\end{array}$ & 5 & 3 & 3 & 3 & 1 \\
\hline Planificación & 9 & $\begin{array}{l}\text { Para la planificación de la } \\
\text { realización del producto } \\
\text { o prestación del servicio } \\
\text { se incluyen aspectos que } \\
\text { integran: }\end{array}$ & 5 & 3 & 3 & 3 & 1 \\
\hline \multirow{2}{*}{$\begin{array}{l}\text { Procesos } \\
\text { relacionados con el } \\
\text { cliente }\end{array}$} & 10 & $\begin{array}{l}\text { Dentro de la definición de los } \\
\text { requisitos para la realización } \\
\text { del producto o prestación } \\
\text { del servicio se consideran los } \\
\text { aspectos relacionados con: }\end{array}$ & 5 & 3 & 3 & 3 & 1 \\
\hline & 11 & $\begin{array}{l}\text { En los procesos de } \\
\text { comunicación con las partes } \\
\text { interesadas se consideran los } \\
\text { aspectos relacionados con: }\end{array}$ & 5 & 3 & 3 & 3 & 1 \\
\hline Diseño y desarrollo & 12 & $\begin{array}{l}\text { Se consideran dentro del } \\
\text { diseño y desarrollo de los } \\
\text { productos/servicios, los } \\
\text { aspectos necesarios para } \\
\text { satisfacer los requisitos de: }\end{array}$ & 5 & 3 & 3 & 3 & 1 \\
\hline Compras & 13 & $\begin{array}{l}\text { En la información de entrada } \\
\text { para realizar las compras se } \\
\text { tienen en cuenta aspectos } \\
\text { relacionados con: }\end{array}$ & 5 & 3 & 3 & 3 & 1 \\
\hline \multirow{2}{*}{$\begin{array}{l}\text { Producción / } \\
\text { prestación del } \\
\text { servicio }\end{array}$} & 14 & $\begin{array}{l}\text { El control de los procesos de } \\
\text { producción/prestación del } \\
\text { servicio integra: }\end{array}$ & 5 & 3 & 3 & 3 & 1 \\
\hline & 15 & $\begin{array}{l}\text { La propiedad del cliente se } \\
\text { garantiza considerando de } \\
\text { forma integrada los aspectos } \\
\text { relacionados con: }\end{array}$ & 5 & 3 & 3 & 3 & 1 \\
\hline
\end{tabular}




\begin{tabular}{|c|c|c|c|c|c|c|c|}
\hline \multicolumn{8}{|c|}{ NIVEL II } \\
\hline \multirow[b]{3}{*}{ DIMENSIONES } & \multirow[b]{3}{*}{$\begin{array}{c}\text { NUEVO } \\
\text { CONTENIDO }\end{array}$} & \multirow[b]{3}{*}{ PREGUNTA } & \multicolumn{5}{|c|}{ ESCALA } \\
\hline & & & A & B & $\mathrm{C}$ & D & $\mathrm{E}$ \\
\hline & & & 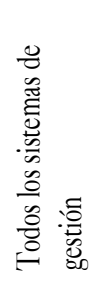 & 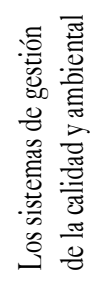 & 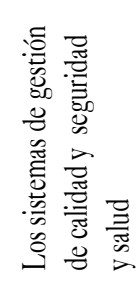 & 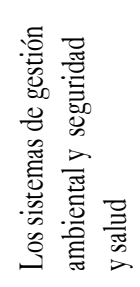 & 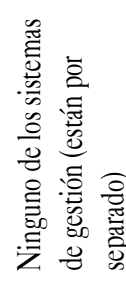 \\
\hline $\begin{array}{l}\text { Producción / } \\
\text { prestación del } \\
\text { servicio }\end{array}$ & 16 & $\begin{array}{l}\text { Para la preservación del } \\
\text { producto o prestación del } \\
\text { servicio se consideran de } \\
\text { forma integrada los requisitos } \\
\text { de: }\end{array}$ & 5 & 3 & 3 & 3 & 1 \\
\hline $\begin{array}{l}\text { Equipos de } \\
\text { medición y } \\
\text { seguimiento }\end{array}$ & 17 & $\begin{array}{l}\text { El control a los equipos de } \\
\text { seguimiento y medición } \\
\text { incluye los de: }\end{array}$ & 5 & 3 & 3 & 3 & 1 \\
\hline No conformidades & 18 & $\begin{array}{l}\text { En el tratamiento de las no } \\
\text { conformidades se integran los } \\
\text { aspectos relacionados con: }\end{array}$ & 5 & 3 & 3 & 3 & 1 \\
\hline $\begin{array}{l}\text { Relación entre } \\
\text { funciones del } \\
\text { personal y los } \\
\text { objetivos de la } \\
\text { organización }\end{array}$ & 19 & $\begin{array}{l}\text { Se encuentran alineadas las } \\
\text { funciones del personal con } \\
\text { los objetivos dentro del } \\
\text { sistema de gestión de la } \\
\text { organización integrando los } \\
\text { aspectos de: }\end{array}$ & 5 & 3 & 3 & 3 & 1 \\
\hline $\begin{array}{l}\text { Responsabilidad y } \\
\text { autoridad }\end{array}$ & 20 & $\begin{array}{l}\text { La definición de autoridad } \\
\text { y responsabilidad incluye } \\
\text { aspectos relacionados con: }\end{array}$ & 5 & 3 & 3 & 3 & 1 \\
\hline $\begin{array}{l}\text { Formación y toma } \\
\text { de conciencia }\end{array}$ & 21 & $\begin{array}{l}\text { Dentro de la organización } \\
\text { se realiza formación y } \\
\text { actividades para la toma de } \\
\text { conciencia considerando los } \\
\text { aspectos relacionados con: }\end{array}$ & 5 & 3 & 3 & 3 & 1 \\
\hline Auditorias & 22 & $\begin{array}{l}\text { Dentro del sistema de } \\
\text { gestión de la organización la } \\
\text { planificación de las auditorías } \\
\text { se realiza incluyendo: }\end{array}$ & 5 & 3 & 3 & 3 & 1 \\
\hline $\begin{array}{l}\text { Revisión por la } \\
\text { dirección }\end{array}$ & 23 & $\begin{array}{l}\text { En la revisión que realiza la } \\
\text { dirección se consideran los } \\
\text { aspectos relacionados con: }\end{array}$ & 5 & 3 & 3 & 3 & 1 \\
\hline
\end{tabular}


Alexandra Bonilla Palacios, Jorge Martínez García

\begin{tabular}{|c|c|c|c|c|c|c|c|}
\hline $\begin{array}{l}\text { Requisitos legales } \\
\text { y otros }\end{array}$ & 24 & $\begin{array}{l}\text { Los requisitos legales y otros } \\
\text { se identifican y evalúan } \\
\text { considerando los aspectos } \\
\text { relacionados con: }\end{array}$ & 5 & 3 & 3 & 3 & 1 \\
\hline Riesgos & 25 & $\begin{array}{l}\text { La identificación y gestión de } \\
\text { los riesgos se realiza teniendo } \\
\text { en cuenta: }\end{array}$ & 5 & 3 & 3 & 3 & 1 \\
\hline $\begin{array}{l}\text { Funciones } \\
\text { del personal / } \\
\text { competencias }\end{array}$ & 26 & $\begin{array}{l}\text { En la definición de las } \\
\text { competencias del personal } \\
\text { de acuerdo a sus funciones se } \\
\text { priorizan los aspectos para: }\end{array}$ & 5 & 3 & 3 & 3 & 1 \\
\hline $\begin{array}{l}\text { Recursos para } \\
\text { el desarrollo y } \\
\text { sostenimiento de } \\
\text { los sistemas de } \\
\text { gestión }\end{array}$ & 27 & $\begin{array}{l}\text { Los recursos humanos, } \\
\text { financieros y físicos se } \\
\text { suministran para el sistema } \\
\text { de gestión de la organización } \\
\text { dando prioridad a: }\end{array}$ & 5 & 3 & 3 & 3 & 1 \\
\hline $\begin{array}{l}\text { Medición, análisis y } \\
\text { mejora }\end{array}$ & 28 & $\begin{array}{l}\text { Se analizan los resultados } \\
\text { de los procesos y se plantean } \\
\text { acciones de forma integrada } \\
\text { considerando: }\end{array}$ & 5 & 3 & 3 & 3 & 1 \\
\hline
\end{tabular}

\begin{tabular}{|c|c|c|c|c|c|c|c|}
\hline \multicolumn{8}{|c|}{ NIVEL III } \\
\hline \multirow{3}{*}{\multicolumn{2}{|c|}{ DIMENSIONES }} & \multirow[b]{3}{*}{ PREGUNTA } & \multicolumn{5}{|c|}{ ESCALA } \\
\hline & & & A & B & $\mathrm{C}$ & $\mathrm{D}$ & $\mathrm{E}$ \\
\hline & & & 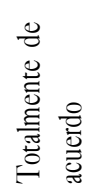 & 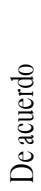 & 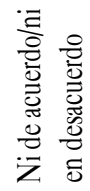 & 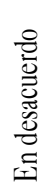 & 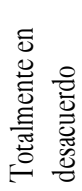 \\
\hline Nivel gerencial & 29 & $\begin{array}{l}\text { La alta dirección define las políticas, } \\
\text { objetivos y metas alineadas con los } \\
\text { sistemas de gestión de calidad, el } \\
\text { ambiental y seguridad y salud. }\end{array}$ & 5 & 3 & 3 & 3 & 1 \\
\hline $\begin{array}{l}\text { Compromiso de la } \\
\text { dirección }\end{array}$ & 30 & $\begin{array}{l}\text { En el desarrollo del sistema integrado } \\
\text { de gestión de la organización se } \\
\text { refleja el compromiso de la alta } \\
\text { dirección. }\end{array}$ & 5 & 3 & 3 & 3 & 1 \\
\hline Eficacia del sistema & 31 & $\begin{array}{l}\text { La mejora continua del sistema } \\
\text { integrado de gestión de la } \\
\text { organización es impulsada desde la } \\
\text { alta dirección. }\end{array}$ & 5 & 3 & 3 & 3 & 1 \\
\hline Comunicación & 32 & $\begin{array}{l}\text { La alta dirección promueve canales } \\
\text { de comunicación que involucra por } \\
\text { completo al sistema integrado de } \\
\text { gestión de la organización. }\end{array}$ & 5 & 3 & 3 & 3 & 1 \\
\hline
\end{tabular}




\begin{tabular}{|c|c|c|c|c|c|c|c|}
\hline \multicolumn{8}{|c|}{ NIVEL III } \\
\hline \multirow{3}{*}{\multicolumn{2}{|c|}{ DIMENSIONES }} & \multirow[b]{3}{*}{ PREGUNTA } & \multicolumn{5}{|c|}{ ESCALA } \\
\hline & & & A & B & C & $\mathrm{D}$ & $\mathrm{E}$ \\
\hline & & & 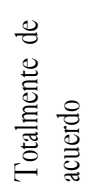 & 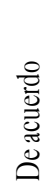 & 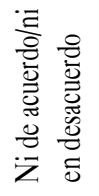 & 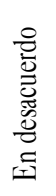 & 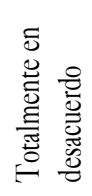 \\
\hline \multirow{3}{*}{ Nivel táctico } & 33 & $\begin{array}{l}\text { Los sistemas de gestión de la calidad, } \\
\text { ambiental y seguridad y salud, } \\
\text { en los niveles intermedios de la } \\
\text { organización se encuentran alineados, } \\
\text { es decir, todos apuntan a un mismo } \\
\text { objetivo. }\end{array}$ & 5 & 3 & 3 & 3 & 1 \\
\hline & 34 & $\begin{array}{l}\text { Los sistemas de gestión de la calidad, } \\
\text { ambiental y seguridad y salud, en los } \\
\text { niveles intermedios de la organización } \\
\text { son compatibles entre ellos. }\end{array}$ & 5 & 3 & 3 & 3 & 1 \\
\hline & 35 & $\begin{array}{l}\text { Los sistemas de gestión de la calidad, } \\
\text { ambiental y seguridad y salud, en los } \\
\text { niveles operativos de la organización } \\
\text { son complementarios entre ellos. }\end{array}$ & 5 & 3 & 3 & 3 & 1 \\
\hline Nivel operacional & 36 & $\begin{array}{l}\text { Para las actividades del nivel } \\
\text { operativo las funciones que se } \\
\text { ejecutan son gestionadas y realizadas } \\
\text { en conjunto considerando los tres } \\
\text { sistemas de gestión. }\end{array}$ & 5 & 3 & 3 & 3 & 1 \\
\hline $\begin{array}{l}\text { Identidad de los } \\
\text { sistemas de gestión }\end{array}$ & 37 & $\begin{array}{l}\text { Los sistemas de gestión de la calidad, } \\
\text { ambiental y seguridad y salud ya no } \\
\text { son reconocidos por separado si no } \\
\text { como un único sistema de gestión } \\
\text { dentro de la organización. }\end{array}$ & 5 & 3 & 3 & 3 & 1 \\
\hline
\end{tabular}

Fuente: elaboración propia.

Se puede concluir que el mecanismo seleccionado y construido es el primero que permite medir el nivel de integración de un sistema de gestión compuesto por las normas ISO 9001:2008, ISO 14001:2004 y OHSAS 18001:2007. Este tendrá que ser expuesto a una muestra representativa de empresa que deje realizar los ajustes finales de instrumentos y definir su generalidad a través de una validación de criterio, ya que el proceso de validación de esta investigación se soportó en un componente teórico y de percepción por parte de un grupo de expertos en la materia. Lo anterior permitió fortalecer dicho instrumento al igual que la prueba piloto para validar su funcionamiento en un ambiente real. No obstante, será necesario revisar y mejorar el mecanismo propuesto a través de su aplicación en una muestra representativa de empresas. 


\section{CONCLUSIONES}

Para encontrar un mecanismo que mida niveles de integración de sistemas integrados de gestión fue necesario referirse a los modelos de excelencia creados por instituciones como EFQM, NIST, JUSE y en Colombia la Corporación Calidad. Lo anterior debido a que estas instituciones realizan evaluaciones exhaustivas de las organizaciones aspirantes a los galardones que ofrecen. Estos mecanismos de evaluación incluyen comités seleccionados y cuestionarios rigurosamente creados para medir la excelencia en la gestión de acuerdo con criterios previamente definidos por cada institución; por esto el mecanismo escogido como el más indicado fue el del cuestionario para medir los niveles de integración en organizaciones con sistemas de gestión integrados.

Teniendo en cuenta la naturaleza exploratoria de esta investigación, se puede concluir que se lograron los objetivos inicialmente propuestos. Del primero de ellos, referido a la identificación del mecanismo que se utilizaría para medir los niveles de integración en sistemas de gestión, se determinó que la mejor forma como se podín obtener resultados era por medio de un instrumento de medición como la encuesta, en la cual, de acuerdo con los aspectos que se evalúan para determinar el nivel de integración de sistemas de gestión, se podía concluir el grado de integración en el que se encontraban los sistemas de gestión dentro de una organización, en cuanto a un puntaje que se obtenía después de aplicarse.

En el segundo objetivo se definió el concepto que abarca en mayor medida lo que es la integración de sistemas de gestión y de la definición de los niveles de integración más idóneos y alineados al concepto de integración seleccionado. Esto para finalmente articularlos a un instrumento como la encuesta, que midiera niveles de integración en organizaciones certificadas en ISO 9001, ISO 14001 y OHSAS 18001. Este hecho requirió una rigurosa búsqueda de la información que se encontraba disponible en las diferentes fuentes bibliográficas, que dio como resultado la selección de un concepto de integración que abarcaba la mayoría de las definiciones encontradas para dar una visión completa de lo que es la integración de sistemas de gestión. Asimismo, se logró identificar la teoría de niveles de integración alineados con el concepto de integración seleccionado, los cuales permiten medir los aspectos relevantes en la integración de sistemas de gestión. También surgieron algunos cambios en la metodología, pues se estructuró y validó el instrumento de medición, la estructuración se realizó alineando el concepto de integración seleccionado, los niveles de integración definidos y las normas de sistemas de gestión, las cuales son objeto de la medición del nivel de integración, para la validación; además, se hizo una validación de contenido con un grupo de expertos y en esta cada uno realizó aportes individuales que luego fueron consolidados y analizados a través de una prueba no probabilística para observar el grado de correlación de sus respuestas. Esto permitió depurar el instrumento y dio como resultado una herramienta con un contenido más suficiente, claro y pertinente. Como consecuencia de la validación se obtuvieron los ítems que finalmente iba a tener el instrumento y con base en ellos se realizó su codificación. Por último, la validación terminó con la aplicación de una prueba piloto para determinar si el instrumento era viable de acuerdo con el propósito para el que fue construido.

Se puede concluir finalmente que el objetivo general de la investigación ha sido alcanzado satisfactoriamente, pues se generó una propuesta de mecanismo inicial para medir niveles de integración que deberá ser aplicado en una muestra de empresas, con el propósito de realizar una validación final del instrumento incluyendo sus últimos ajustes. Por ende, podríamos decir que esta investigación ha suministrado un primer aporte 
para las instituciones académicas que quieran ahondar en este tipo de mecanismo.

Se recomienda a futuros investigadores que tengan interés en aplicar el instrumento de medición aquí propuesto de manera masiva que realicen una validación más profunda de este aplicándolo en una muestra más grande de organizaciones reales para verificar la consistencia en la información que se genera de dicho instrumento y poder garantizar en mayor medida su confiablidad. Al hacer esto, podrá ser utilizado para analizar la información de manera generalizada de un sector de la industria; todo esto teniendo en cuenta los parámetros definidos en el instructivo diseñado para la aplicación del instrumento, en la que se detalla el concepto de integración adoptado, la teoría de nivel de integración, las escalas de medición, la forma de administración, los criterios de selección de las empresas, el envío del instrumento, el análisis de la información y la retroalimentación de la medición. Adicionalmente, se recomienda realizar un contacto directo con el responsable del sistema de gestión en la organización, que será el que dará respuesta al instrumento con el fin de comunicarle de primera mano los beneficios que trae consigo un sistema de gestión integrado y la importancia de identificar el nivel de integración donde que se encuentra.

\section{REFERENCIAS}

Amaya, R. (1993). Diagnóstico pedagógico: fundamentos teóricos. Oviedo: Universidad de Oviedo.

Asociación Española de Normalización y Certificación [AENOR] (2005). Guía para la Integración de los Sistemas de Gestión. UNE 66177. Madrid: autor.

Babativa, G. y Corzo, J. (14 de mayo de 2013). Métodos no paramétricos. Pruebas de hipótesis en problemas de dos y K muestras [diapositivas]. Bogotá: Universidad Santo Tomás.

Beckmerhagen, I., Berg, H., Karapetrovic, S., Willborn, W. (2003). Integration of management systems: focus on safety in the nuclear industry. International Journal of Quality \& Reliability Management, 20(2), 210-228.

Bernardo, M. (2009). Integracio de sistemes estandarditzats de gestio: una analisis empírica (Tesis de doctorado). Girona, Universitat de Girona.

Bernardo, M., Casadesus, M., Karapetrovic, S. y Heras, I. (2009). How integrated are environmental, quality and other standardized management systems? An empirical study. Journal of Cleaner Production, (17), 742-750.

British Standard Institution [BSI] (2008). Specification of common management system requirements as a framework for integration. Publicly Available Specification PAS 99. London: BSI.

Bucci, N. (1 de febrero de 2011). Análisis del sistema integrado de gestión para las organizaciones desde la perspectiva sistémica. Revista Digital de Investigación y postgrado, 1(1), 83-97.

Casadesús, M. (1999). La normativa de aseguramiento de la calidad ISO 9000. Girona: Universidad de Girona.

Casadesus, M. Karapetrovic, S. (7-9 de septiembre de 2011). Integrating Management Systems: A dynamic study of Spanish firms. En 5th International Conference on Industrial Engineering and Industrial Management, XV Congreso de Ingeniería de Organización. Cartagena, Colombia.

Casadesus, M., Karapetrovic, S. y Heras, I. (2009). Sistemas de gestión estandarizados: ¿existen 
sinergias?. Revista Europea de Dirección y Economía de la Empresa, 18(2), 161-174.

Castillo P., D. y Martínez, J. C. (2010). Enfoque para combinar e integrar la gesstión de sistemas (2da. Ed.). Bogotá: Icontec.

Celis, G. y Carmen, R. (2012). Sistema Integrado de Gestión de la Calidad e Higiene y Salud Ocupacional para la industria farmacéutica Venezolana. Caracas: Universidad Católica Andrés Bello.

Corporación Calidad. (2012). Modelo de excelencia para organizaciones de clase mundial. Bogotá: Ministerio de Comercio Industria y Turismo.

Crosby, P. (1979). Quality is free: the art of making quality certain. Nueva York: McGraw-Hill.

Crosby, P. (1987). La calidad no cuesta. Madrid: Díaz de Santos.

Dale, B. (2003). Managing quality. Malden: Blackwell Publishing Ltd.

Deming, E. y Walton, M. (1990). El método Deming en la práctica. New York: Putman’s Songs.

Deming, W. (1986). Out of the crisis, Massachusetts Institute of Technology. Cambridge: Center for Advanced Engineering Study.

Gómez M., M. (2006). Introducción a la metodología de la investigación cientifica. Córdoba: Brujas.

Griffith, A. y Bhutto, K. (2008). Improving environmental performance through integrated management systems (IMS) in the UK. Management of Environmental Quality: An International Journal, 19(5), 565-578
Hernández, Fernández y baptista. (2010). Metodología de la investigación. 5.a edición. México: Mc Graw Hill.

Hernández, M. E. (2007). Propuesta metodológica de integración de sistemas de seguridad e higiene, medio ambiente y calidad para fabricantes de detergentes en México. México, D. C.: Universidad de La Salle.

Internacional Organization for Standardization. (2008). The integrated use of management system standards. Ginebra: ISO

Internacional Organization for Standardization [ISO] (2010). High-level structure and identical text for management system standards and common core management system terms and definitions. DRAFT ISO GUIDE 83. Ginebra: ISO.

Internacional Organization For Standardization [ISO] (s. f.). ISO, ISO 14000. Recuperado de https:// www.iso.org/iso-14001-environmental-management.html

Jonker, J., Karapetrovic, S. (2004). Systems thinking for the integration of management systems. Business Process Management Journal, 10(6), 608-615.

Jørgensen, T. (2008). Towards more sustainable management systems: through life cycle management and integration. Journal of Cleaner Production, 16(10), 1071-1080.

Juran, J. (1951). Quality-control handbook. Nuva York: McGraw-Hill, Book Co.

Juran, J., Gryn, F. y Bingham, R. (1990). Manual de control de la calidad (2da. Ed.). Madrid: Reverté.

Karapetrovic, S. (2003). Musings on integrated management systems. Measuring Business Excelencia, 7(1), 4-13. 
Karapetrovic, S. y Willborn, W. (1998). Integration of quality and environmental management systems. The TQM Magasine, (3), 204-213.

Karapetrovic, S. (2002). Strategies for the integration of management systems and standards. The TQM Magazine, 14(1), 61-67.

Karapetrovic, S. y Jonker, J. (2003). Integration of Standardized management systems: searching for a recipe and ingredients. Total Quality Management ¿̊ Business Excellence, 14(4), 451-459.

Karapetrovic, S. y Willborn, W. (1998). Integration of quality and environmental management systems. The TQM Magasine, 10(3), 204-213.

Kirkby, A. (2002). The one-stop shop. Quality world, $10,2-4$.

Labodová, A. (2004). Implementing integrated management systems using a risk analysis based approach. Journal of Cleaner Production, 12(6), 571-580.

Macgregor Associates (1996). Study on Management System Standards. Londres: British Standards Institute.

Moreno, J. (2007). Guía para la aplicación del modelo EFQM en entidades de acción social, Cuaderno de gestión (2da. Ed). Madrid: Fundación Luis Vives.

National Institute of Standards and Technology [NIST] (s. f.). Bladrige Performance Excellence Program - Malcolm Baldrige National Quality Award. U.S.A. Recuperado de https://www.nist.gov/ baldrige.

Nieves. C. y McDonnell. L. (7 y 8 de septiembre de 2006). Comparación entre los modelos de gestión de Calidad Total. En X Congreso de Ingeniería de Organización, Valencia, España.

Pojasek, R. (2006). Is your Integrated Management System Really Integrated? Environmental Quality Management, 1(2), 89-97.

Salgado, R. A. (2010). Sistema integrado de gestión para la construcción de obras civiles, aplicado a la construcción de puentes. Valdivia Universidad Austral de Chile.

Seddon, J. (1997). Ten arguments against ISO 9000. Managing Service Quality: An International Journal, 7(4), 162-168.

Seghezzi, H. (1997). Business concept redesign. Total Quality Management, 8(2), 42-49.

Tejada, F. y Peña, G. (2009). Reflexiones sobre las características constitutivas de la gestión integral. Signos, 1, 79-93.

The W. Edwards Deming Institute (s. f.). Deming Prise. Recuperado de https://deming.org/deming-theman/photographs/all/deming-prize-medal-1045

Wilkinson, G. y Dale, B. (1999). Integration of quality, environmental and health and safety management systems: an examination of the key issues. Journal of Engineering Manufacture, 213(3), 275-283.

Zeithaml, V., Parasuraman, A. y Berry, L. (1985). Problems and Strategies in Services Marketing. Journal of Marketing, 49(2), 33-46.

Zeng, S., Shi, J. y Lou, G. (2007). A synergetic model for implementing an integrated management system: an empirical study in China. Journal of Cleaner Production, 15(18), 1760-1767. 